\title{
SPECTRAL PRE-ADAPTATION FOR TWO-STEP ARBITRARY-SHAPE-SUPPORT IMAGE RESTORATION
}

\author{
Chaoqun Dong \\ Dept. of Electronic \& Computer Engineering \\ HKUST, Hong Kong
}

Javier Portilla*

Instituto de Óptica

CSIC, Madrid

\begin{abstract}
In recent years, boundary problems associated to image restoration have attracted the attention of imaging scientists. We explore here the scenario of arbitrary boundary shapes (exemplified by blurred-background images), starting from a general discussion and advancing towards realist conditions, with simulations and real photographic images. We describe our Spectral-Pre-Adaptation (SPA) method, and compare it to the highly successful unconstrained boundary conditions Alternating Direction Method of Multipliers (UBC ADMM) method. Preliminary results indicate that SPA, combined with highly efficient restoration algorithms, such as Constrained Dynamic (ConDy) L2-relaxed L0, may set the new state-ofthe-art (in performance and computational efficiency) for this kind of problems.
\end{abstract}

Index Terms - image restoration; arbitrary boundary conditions; spectral models; extrapolation; circular boundary condition problem

\section{INTRODUCTION}

Virtually all image restoration methods (and among them, those providing current state-of-the-art, e.g., [1, 2, 3]) rely on the Discrete Fourier Transform (DFT) for some intermediate linear compensation steps within globally non-linear algorithms. However, a well-known problem of DFT is that it assumes unnatural circular boundary conditions (CBCs) on the image. This provokes that excellent results obtained on simulations do not hold acceptable when translated to CBC-free images (either realistic simulations or real images). Our motivation to attack the boundary problem is, in addition, to restore inhomogeneously blurred images having uniformly convolved areas with arbitrarily-shaped boundaries (because of local movement, defocus, etc.).

Some of the new methods proposed to deal with boundaries are adaptations of previous restoration algorithms [4, 5, 6]. Some others follow a different, two-step, strategy: given a non-CBC blurred image observation, first to process the observation to make it compatible with $\mathrm{CBCs}$, and then to

\footnotetext{
*Funded by the Spanish Government grant FIS2016-75891-P.
}

apply any standard (i.e., CBC-assuming) restoration method. Among the latter, classical ones are window masking (analogous to spectral estimation methods, see e.g. [7]), and simple image extrapolation, either by reflection or by using an "edge taper" filter forcing smoothness in the toroidal (CBC) support (see "edgetaper.m" (R)Matlab function). Window masking is far from ideal, as it causes serious information loss along the boundaries. This loss becomes unacceptable when making the window mask very smooth, to better respect the original spectrum. Reflection is severely limited to images without obliquely oriented spatial features being blurred with $\mathrm{x}$ - and y-symmetrical kernels [8]. Edge tapering is usually better than reflection, but not yet satisfactory $[8,5]$. Furthermore, none of these classical recipes can be easily adapted to generic unconstrained boundary shapes.

Within the two-step approach, Reeves [9] proposed an elegant boundary extension to obtain a CBC-seamless image, by means of a Laplacian regularizer. In [8] we used the blurring kernel information to estimate not just a CBC-seamless image, but one also likely in terms of being the output of the blurring kernel (plus noise). Later on, the same idea was applied to interpolate samples in aliased blurred observations to reduce restoration artifacts [10]. Here we apply the same Spectral Pre-Adaptation (SPA) approach to deal with arbitrarily shaped boundaries, with direct application, among others, to process images with a sharp foreground plane on a blurred background, and compare results with the state-of-the-art [5].

\section{SPECTRAL PRE-ADAPTATION METHOD}

\subsection{Problem formulation}

Let us consider an observation $\mathbf{y}$, made of $K$ areas, each convolved with a different kernel, and distributed on arbitrarily shaped non-overlapping sub-images $\left\{u_{k}, k=1 \ldots K\right\}$. These sub-images can be, e.g., a far out-of-focus background, a moving object, etc. Our goal is to deconvolve each of these areas. In this paper we will show examples of $K=2$, being $u_{1}$ a blurred background, and $u_{2}$ the rest, a non-degraded foreground image. $u_{k}$ can be thought of as part of an extended image $z_{k}$, a rectangular array of pixels CBC-convolved with a blurring kernel $h_{k}$. 
Given an arbitrary lexicographical vector reordering we can express $\mathbf{u}_{k}=\mathbf{S}_{k} \mathbf{z}$, where $\mathbf{S}_{k}$ is a $N_{k} \times M$ matrix, with $M>N_{k}$, acting as a mask to select only those $N_{k}$ pixels of $z_{k}$ belonging to $u_{k}$. Following a standard stationary Gaussian model, we can express the minus log-likelihood of the extended image $Z_{k}$ in the frequency domain as:

$$
-\log \left(p\left(Z_{k}(u, v)\right) \propto \sum_{i, j} \frac{\left|Z\left(u_{i}, v_{j}\right)\right|^{2}}{P_{Z_{k}}\left(u_{i}, v_{j}\right)}+C,\right.
$$

where $C$ is a constant and $P_{Z_{k}}$ represents the power spectral density (PSD) of $z_{k}$, the so-extended $u_{k}$ sub-image, with

$$
\begin{aligned}
P_{Z_{k}}(u, v) & =\mathbb{E}\left|Z_{k}(u, v)\right|^{2} \\
& =\left|H_{k}(u, v)\right|^{2} \mathbb{E}|X(u, v)|^{2}+\mathbb{E}|W(u, v)|^{2} \\
& =\left|H_{k}(u, v)\right|^{2} P_{X}(u, v)+\sigma_{W}^{2} .
\end{aligned}
$$

Here the upper case letters represent the signals and functions (the ideal image $x$, the kernels $h_{k}$ and the noise $w$ ) in the Fourier space, and we have assumed, for simplicity, uniform additive white noise (although to be white is not a constraint for the noise in our method). For $P_{X}$ we use a separable auto-regressive 1-tap model with parameters $\sigma_{x}=30$ (adjusted to the $[0,255]$ range) and correlation factor $\rho=$ 0.65 [8]. Note that the frequencies having more weight in the cost functions are those with low $P_{Z_{k}}$ values, typically corresponding to the zeros of $H_{k}$. Keeping these frequencies suppressed (or heavily damped) in the extended image spectrum is what makes SPA essentially different from mere smooth inter/extrapolation.

Our goal is to minimize the cost function of Eq.(1) subject to $\mathbf{S}_{k} \mathbf{z}_{k}=\mathbf{u}_{k}$ (consistency with the observation $\mathbf{u}_{k}$ ), that is:

$$
\hat{\mathbf{z}}_{k}\left(\mathbf{u}_{k}\right)=\arg \min _{\mathbf{z}}\left\|\mathbf{D}_{P_{Z_{k}}}^{-1 / 2} \mathbf{F}^{*} \mathbf{z}\right\|^{2} \text {, s.t. } \mathbf{S}_{k} \mathbf{z}=\mathbf{u}_{k},
$$

where $\mathbf{F}^{*}$ is a matrix performing a $2 \mathrm{D}-\mathrm{DFT}$, and $\mathbf{D}_{P_{Z_{k}}}$ is a diagonal matrix whose entries correspond to $P_{Z_{k}}(u, v)$.

\subsection{An efficient solution}

Eq. (3) can be reformulated to an unconstrained optimization [8], yielding:

$$
\hat{\mathbf{z}}\left(\mathbf{u}_{k}\right)=-\left(\mathbf{E}_{\mathbf{k}} \mathbf{F} \mathbf{D}_{P_{Z}}^{-1} \mathbf{F}^{*} \mathbf{E}_{\mathbf{k}}^{T}\right)^{-1} \mathbf{E}_{\mathbf{k}} \mathbf{F} \mathbf{D}_{P_{Z}}^{-1} \mathbf{F}^{*} \mathbf{S}_{\mathbf{k}}^{\mathbf{T}} \mathbf{u}_{k},
$$

where $\mathbf{E}_{\mathbf{k}}^{T}\left(M \times\left(M-N_{k}\right)\right)$ performs the extension of the estimated pixels to a vector containing the whole image and having set the observed pixels to zero. $\mathbf{S}_{\mathbf{k}}{ }^{T}\left(M \times N_{k}\right)$, conversely, preserves the observed pixels, and sets to zero the rest. Their transpose do the reverse (selection) operations. For attacking the matrix inversion problem of Eq. (4) (note that the involved matrix is not shift-invariant), we call $\mathbf{A}=$ $\mathbf{E}_{\mathbf{k}} \mathbf{F} \mathbf{D}_{P_{Z}}^{-1} \mathbf{F}^{*} \mathbf{E}_{\mathbf{k}}{ }^{T}$ and $\mathbf{b}\left(\mathbf{u}_{k}\right)=-\mathbf{E}_{\mathbf{k}} \mathbf{F} \mathbf{D}_{P_{Z}}^{-1} \mathbf{F}^{*} \mathbf{S}_{\mathbf{k}}^{\mathbf{T}} \mathbf{u}_{k}$. First we use Neumann's series [11] $\mathbf{A}^{-1}=\sum_{n=0}^{\infty}(\mathbf{I}-\mathbf{A})^{n}$ to obtain the iterative method solving $\hat{\mathbf{z}}\left(\mathbf{u}_{k}\right)=\mathbf{A}^{-1} \mathbf{b}\left(\mathbf{u}_{k}\right)$ :

$$
\hat{\mathbf{z}}^{(n+1)}=\hat{\mathbf{z}}^{(n)}+\lambda\left(\mathbf{b}\left(\mathbf{u}_{k}\right)-\mathbf{A} \hat{\mathbf{z}}^{(n)}\right) .
$$

We have used $\lambda=2 \sigma_{w}^{2}$ as an approximation to $\lambda=2 /\|\mathbf{A}\|$. Secondly, we have used the Minimal Polynomial Extrapolation $[10,12,13]$ for accelerating the convergence of $\hat{\mathbf{z}}$ in Eq. (5). This method performs a linear regression for the $Q$ consecutive differences in the vector sequence of Eq. (5). A simple manipulation allows to compute the limit of the series from the linear predictor $[12,13]$. This process is repeated $P$ times, until getting close enough to convergence.

\subsection{Initial extrapolation/interpolation guess}

In practice, starting from a guess not too far from the final solution may greatly reduce the number of required iterations $P$. Here we propose to use a smart shift-variant inter/extrapolation filtering for obtaining a suitable initial guess. Naming $m_{k}(x, y)$ the mask on an rectangular image support including $\mathbf{u}_{k}$ indicating which pixels belong to $\mathbf{u}_{k}$ (1s) and which are unknown (0s), we obtain a first prediction of $z_{k}(x, y)$ through:

$u_{k}^{e x t}(x, y)=\frac{h_{e}(x, y) *\left(m_{k}(x, y) y(x, y)\right)}{h_{e}(x, y) * m_{k}(x, y)}$

$z_{k}^{(0)}(x, y)=\left(1-m_{k}(x, y)\right) u_{k}^{e x t}(x, y)+m_{k}(x, y) y(x, y)$

The estimation is an average of known pixels giving more weight to the closer pixels and less weight to the farther ones. This is achieved by convolving the masked observation with an inter/extrapolation kernel $h_{e}$ that is a decreasing function of its radius. However, for the involved sum to be an average it is necessary to divide it by the number of involved known pixels, that is, by convolving $m_{k}(x, y)$ with $h_{e}$.

In addition, in order to obtain a reasonable estimate, $h_{e}$ should not blur more than necessary. At short distance to known pixels it must effectively behave like a small filter, whereas at locations far from known pixels it must behave like a large filter. An elegant solution is to choose a large radially symmetric filter with values falling off very quickly, being peaky at the center and rapidly flattening. Normalization does the rest. We have chosen $h_{e}(r)=r^{-n_{e}}$, for $r>0$, and $h_{e}(0)=0$, with the exponent $n_{e}=7$ chosen to be the highest value not producing numerical instability.

\subsection{Extending the original support}

By enlarging the estimated area to go beyond the original support of the image it has been shown that one can effectively recover pixels outside the original image support, within half of the kernel size extension $[4,5,8]$. However, it may be interesting to extend the image even farther. The reason is that, due to we are using a discrete representation, zeros of $h_{k}$ may fall on in-between discrete samples in the Fourier domain. To take full advantage of these zeros in the estimation process, it is convenient to avoid that whenever is possible. In separable filters, zeros are aligned and repeated by rows and columns, 
which makes this effect more relevant. This can be achieved by choosing an extension of

$$
L_{x}^{e}=\left(\left\lceil D_{x} / n_{x}^{(k)}\right\rceil n_{x}^{(k)}-D_{x}\right) / 2,
$$

being $D_{x}$ and $n_{x}^{(k)}$ the size of the image and of the kernel $h_{k}$, respectively (in the horizontal direction, same in vertical). This makes the new image dimensions $D+2 L^{e}$ to be integer multiple of $n^{(k)}$. More generally, we may want to choose the image support size $\left(D_{y}, D_{x}\right)$ for the $z_{k}$ including $u_{k}$ that minimizes the entropy rate of $p_{z_{k}}$, by minimizing $\left\langle\log P_{Z_{k}}(u, v)\right\rangle_{(u, v)}[14]{ }^{1}$

\section{EXPERIMENTS, RESULTS AND DISCUSSION}

\subsection{An illustrating academic experiment}

In [8] we extended the boundaries of non-CBC observed images so that they could be safely restored using standard methods. Is the same approach also suitable for accurately inpainting blurred images, so that they can be safely restored afterwards? In [5] a beautiful (and somehow surprising) result was shown: relatively large areas of lost pixels of a highly blurred image (a uniform $19 \times 19$ filter was used, with very low noise, $B S N R=60 \mathrm{~dB}$ ) were recovered by using a unconstrained boundary condition (UBC) variant of the ADMM restoration algorithm (for generic ADMM see, e.g., [15]). In Figure 1 we show the simulated observation (panel (a)), and the impressive result Almeida and Figueiredo obtained with their method (panel (c)). We also show our blur in-painting result using SPA ( $L^{e}=5$, computed using Eq. (7), $Q=25$, and $P=500$ ) (panel b), and the result of restoring (b) with the same method, but without unknown-pixel mask (panel (d)). The fact that both results are similar indicates that $\mathrm{s}$ simple spectral method (SPA) may suffice to regenerate incomplete blurred observations.

\subsection{Blurred background image simulation}

In Figure 2 we present the results of an image simulation composed by an object in focus (a hand, foreground) partially occluding a blurry background (the standard $512 \times 512$ pixel "Pirate"). We used a uniform disk of diameter 9 pixels for simulating a defocus, and $\sigma_{w}=0.3$. Here we applied $L_{2}$ relaxed- $L_{0}$ ConDy-10 [2] to restore the SPA result of panel (b), see panel (d). We compare again with UBC ADMM (FAMD variant) [5] ${ }^{2}$. We can see how both methods provide excellent results. Under more careful inspection, though, one can see that our combined method has better preserved texture. This is a feature of $L_{2}$-relaxed $L_{0}$-sparse methods, as compared to TV-like methods. It also has less artifacts (see pirate's chin). Not surprisingly, our two-step method obtains

\footnotetext{
${ }^{1}$ SPA $®$ Matlab code available in $®$ researchgate.net, upon publication.

${ }^{2}$ We thank Almeida and Figueiredo for making their code available.
}

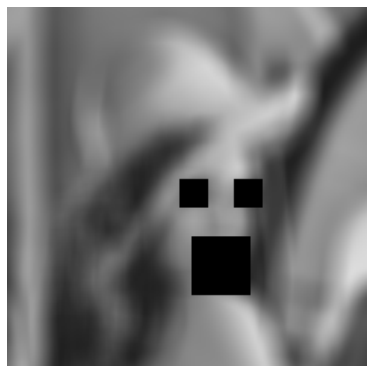

(a)

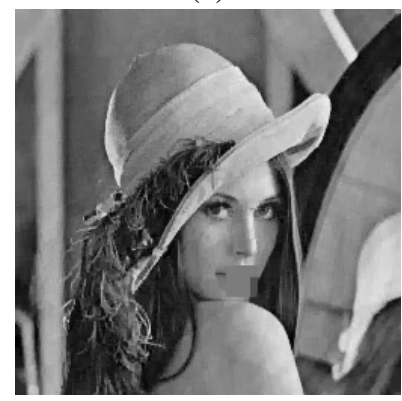

(c)

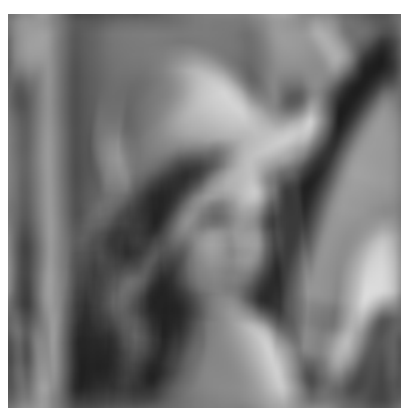

(b)

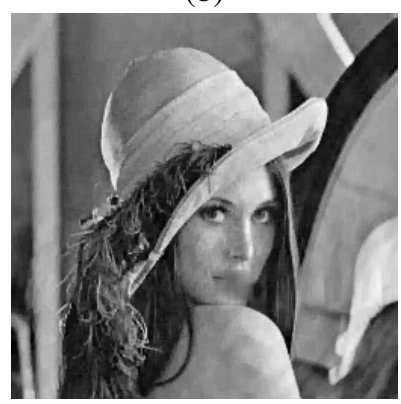

(d)
Fig. 1. Recovering lost regions (panel (a)) in blurred images, using UBC ADMM [5] (panel (c)), vs. using SPA in-painting (panel b) followed by standard restoration (panel (d)).

a significantly better Signal-to-Noise ratio result on the background $(24.95 \mathrm{~dB}$ vs. $24.44 \mathrm{~dB})$. In this case the improvement comes from the restoration method, because using SPA plus "unmasked" UBC ADMM we obtain very close results (24.47 dB) than with "masked" ADMM. In this experiments we used 150 iterations for UBC ADMM ("masked" and "unmasked"), close to convergence, and used $Q=25, L^{e}=8$ and $P=4$ for SPA. Computation times in a 2 x $2,26 \mathrm{GHz}$ Quad-Core Intel Xeon using ${ }^{\circledR}$ Matlab code was ca. $80 \mathrm{~s}$. for both UBC ADMM (with and without mask), 4 times longer than the sequence of SPA (ca. $6 \mathrm{~s}$.) and $L_{2}-\mathrm{r}-L_{0}$ (ca. $14 \mathrm{~s}$,).

\subsection{Real examples}

First example is a $512 \times 512$ crop of a real picture of a comb in the foreground and a text on the background. It was taken ${ }^{3}$ using a professional camera ${ }^{4}$, indoors, with tripod, and without flash. We took the average of the color channels to convert it into gray scale, so further reducing the noise. In a real image, the foreground must be segmented, and the blurring kernel plus the noise variance must be estimated. In this case none of these requirements were too difficult. First, the foreground is quite dark, compared to the background. To estimate the blurring kernel we assumed a uniform disk, and,

\footnotetext{
${ }^{3}$ Many thanks to Isabel Portilla for kindly providing us with this picture.

${ }^{4}$ Canon EOS 5D Mark II, 20 Mpix, CR2 to uint16 TIFF, focal length $50 \mathrm{~mm}$, exposure $1 / 2500 \mathrm{~s}$., ISO $100, \mathrm{f} / 3.5$
} 


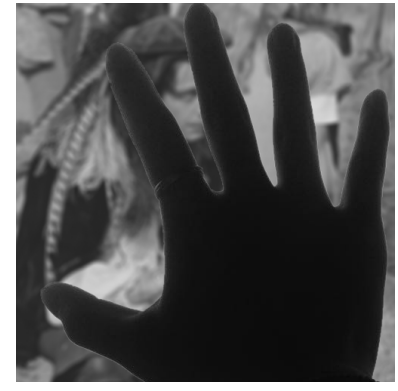

(a)

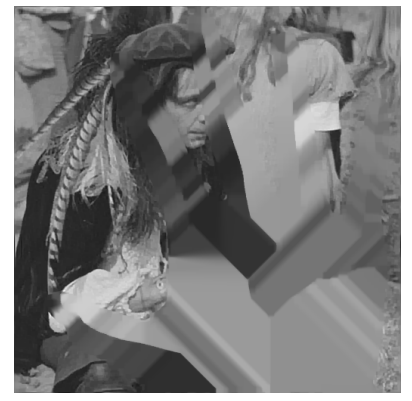

(c)

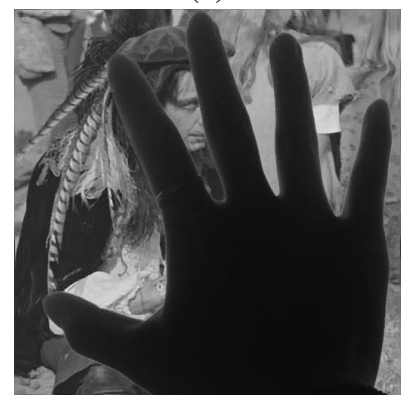

(e)

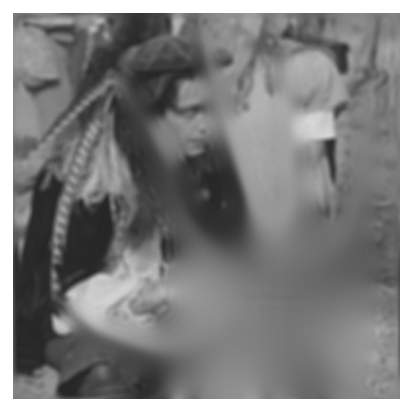

(b)

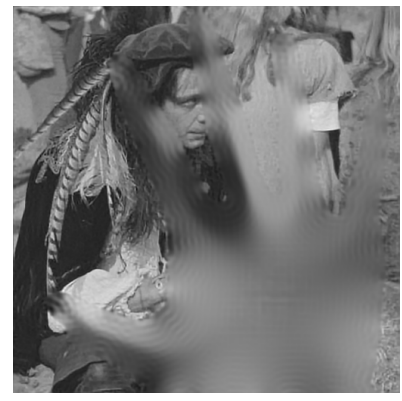

(d)

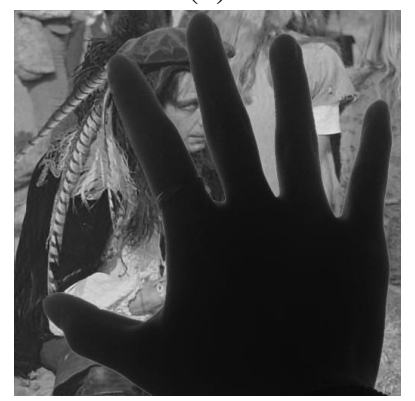

(f)
Fig. 2. (a) Simulated observation; (b) SPA in-painting result; (c) masked ADMM result; (d) $L_{2}-r-L_{0}$ restoration on (b); (e) final masked ADMM result; (f) final SPA $+L_{2}-\mathrm{r}-L_{0}$ result.

from an only-background crop of the same picture, we optimize the fit for the diameter, which turned out to be $D \approx 29.5$. For the noise, we estimate $\sigma_{w} \approx 4$ (note that the range now is $[0,65536])$. Second restoration case is more challenging: a $512 \times 512$ crop from a picture taken with a mobile phone indoors with indirect natural light, without tripod, in JPEG format ${ }^{5}$. The foreground is part of a pen, and the background is a printed text, as before. We obtained the mask through high-pass filtering and morphological operations, and estimated $D \approx 14.5, \sigma_{w} \approx 1.25$ on an only-background subcrop. For the SPA method we used $L^{e}=5, Q=25$, and $M=3$ for both pictures. For the $L_{2}$-relaxed $L_{0}$ restoration we imposed the range $[30000,54000]$ in the comb image and $[120,210]$ for the pen during the estimation loop. In all experiments we used $N_{\text {iter }}=10$ iterations (ConDy-10).

\footnotetext{
${ }^{5}$ 2Mpix camera from Sony Xperia D2203, focal length $2.78 \mathrm{~mm}$ f/2.4, exposure time 1/250 s, JPEG compress: approx. 2.4 bits per RGB pixel.
}

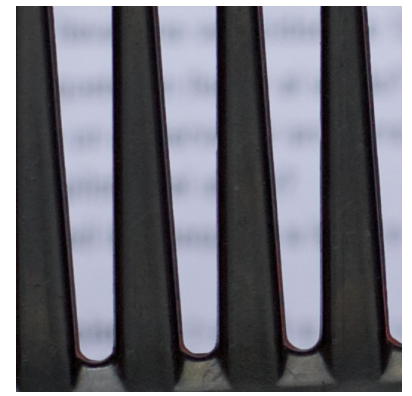

(a)

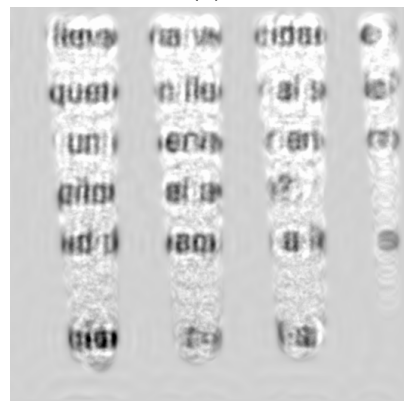

(c)

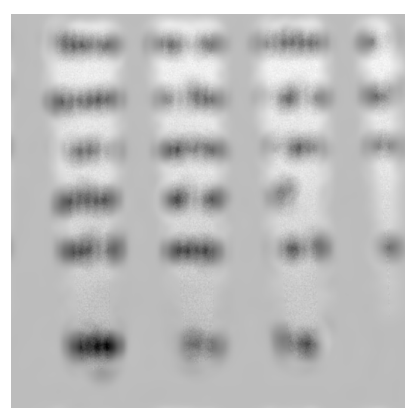

(b)

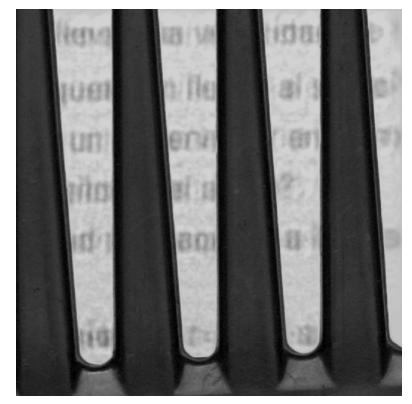

(d)
Fig. 3. Restoring a high quality photography crop. (a) observation; (b) Background SPA result (scaled); (c) Restored background (scaled); (d) Final result

\section{CONCLUSIONS}

We presented a new approach to restore arbitrary-shape blurred images, based on the Spectral Pre-Adaptation method, previously used for tackling circular boundary conditions and aliasing in restoration problems. First, in an academical example we have shown how SPA is able to accurately in-paint heavily blurred images. In a more realistic simulation, our method provided better results than current state of the art. Finally, we successfully applied it to real photographies with out-of-focus background, including one casually captured with a mobile phone camera. The proposed pre-processing allows for an open choice of the restoration method, and this finally translates into better and faster restoration results.
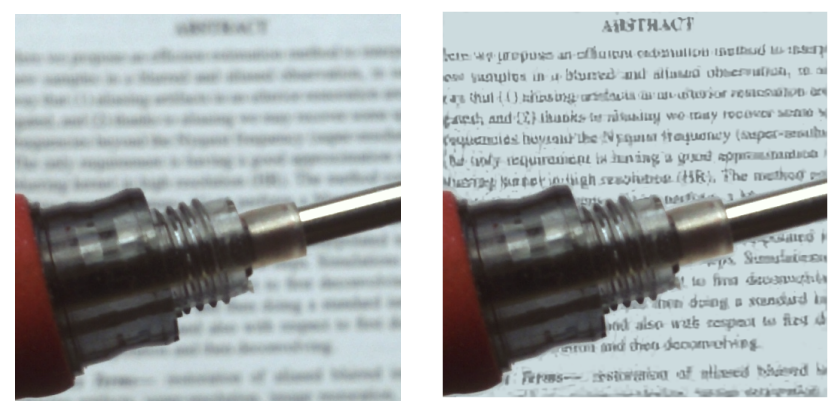

Fig. 4. Restoring a JPEG-compressed medium quality photography. See text for details. 


\section{REFERENCES}

[1] A. Danielyan, V. Katkovnik, and K. Egiazarian, "Bm3d frames and variational image deblurring," IEEE Transactions on Image Processing, vol. 21, no. 4, pp. 1715-1728, April 2012.

[2] J. Portilla, A. Tristan-Vega, and I.W. Selesnick, "Efficient and robust image restoration using multiple-feature L2-relaxed sparse analysis priors," Image Processing, IEEE Transactions on, vol. 24, no. 12, pp. 5046-5059, Dec 2015.

[3] W. Dong, L. Zhang, G. Shi, and X. Wu, "Image deblurring and super-resolution by adaptive sparse domain selection and adaptive regularizations," IEEE Trans. Image Process., vol. 20, no. 7, pp. 1838-1857, 2011.

[4] M. Sorel, "Removing boundary artifacts for real-time iterated shrinkage deconvolution," IEEE Transactions on Image Processing, vol. 21, no. 4, pp. 2329-2334, April 2012.

[5] M. S. C. Almeida and M. Figueiredo, "Deconvolving images with unknown boundaries using the alternating direction method of multipliers," IEEE Transactions on Image Processing, vol. 22, no. 8, pp. 3074-3086, Aug 2013.

[6] A. Matakos, S. Ramani, and J. A. Fessler, "Accelerated edge-preserving image restoration without boundary artifacts," IEEE Transactions on Image Processing, vol. 22, no. 5, pp. 2019-2029, May 2013.

[7] A. Papoulis and S.U. Pillai, Probability, random variables, and stochastic processes, McGraw-Hill electrical and electronic engineering series. McGraw-Hill, 2002.

[8] J. Portilla, "Maximum likelihood extension for non-circulant deconvolution," in Image Processing (ICIP), 2014 IEEE International Conference on, Oct 2014, pp. 4276-4279.

[9] S. J. Reeves, "Fast image restoration without boundary artifacts," IEEE Transactions on Image Processing, vol. 14, no. 10, pp. 1448-1453, Oct 2005.

[10] C. Dong and J. Portilla, "Maximum likelihood interpolation for aliasing-aware image restoration," in 2016 IEEE International Conference on Image Processing (ICIP), Sept 2016, pp. 564568.

[11] G.W. Stewart, Matrix Algorithms: Volume 1: Basic Decompositions, Society for Industrial and Applied Mathematics, 1998.

[12] S. Cabay and L. W. Jackson, "A polynomial extrapolation method for finding limits and antilimits of vector sequences," SIAM Journal on Numerical Analysis, vol. 13, no. 5, pp. 734752, 1976.

[13] Sidi A., Ford W.F., and Smith D. A., "Acceleration of convergence of vector sequences," SIAM Journal on Numerical Analysis, vol. 23, no. 1, pp. 178-196, 1986.

[14] S. Ihara, Information Theory for Continuous Systems, Series on Probability and Statistics. World Scientific, 1993.

[15] R. Glowinski, S.J. Osher, and W. Yin, Splitting Methods in Communication, Imaging, Science, and Engineering, Scientific Computation. Springer International Publishing, 2017. 